\title{
Flicker noise in degenerately doped Si single crystals near the metal-insulator transition
}

\author{
A K RAYCHAUDHURI, ARINDAM GHOSH ${ }^{\dagger}$ and SWASTIK KAR \\ Department of Physics, Indian Institute of Science, Bangalore 560 012, India \\ $\dagger$ Present address: Cavendish Laboratory, University of Cambridge, Madingley Road, \\ Cambridge, UK
}

\begin{abstract}
In this paper we report some of the important results of experimental investigations of the flicker noise near the metal-insulator (MI) transition in doped silicon single crystals. This is the first comprehensive work to study low-frequency noise in heavily doped Si over an extensive temperature range $(2 \mathrm{~K}<T<500 \mathrm{~K})$. The measurements of conductance fluctuations (flicker noise) were carried out in the frequency range $10^{-2}<f<4 \times 10^{1} \mathrm{~Hz}$ in single crystalline $\mathrm{Si}$ across the MI transition by doping with phosphorous and boron. The magnitude of noise in heavily doped $\mathrm{Si}$ is much larger than that seen in lightly doped $\mathrm{Si}$ over the whole temperature range. The extensive temperature range covered allowed us to detect two distinct noise mechanisms. At low temperatures $(T<100 \mathrm{~K})$ universal conductance fluctuations (UCF) dominate and the spectral dependence of the noise is determined by dephasing the electron from defects with two-levels (TLS). At higher temperatures $(T>200 \mathrm{~K})$ the noise arises from activated defect dynamics. As the MI transition is approached, the $1 / f$ spectral power, typical of the metallic regime, gets modified by the presence of discrete Lorentzians which arise from generation-recombination process which is the characteristic of a semiconductor.
\end{abstract}

Keywords. $1 / f$ noise; doped $\mathrm{Si}$; MI transition.

PACS Nos 72.70.+m; 72.80.Cw; 72.80.Ng

\section{Introduction}

Electronic transport in disordered solids has been a topic of considerable interest for decades $[1,2]$. Scaling theories and related developments both in experimental and theoretical fronts have contributed a lot to our recent understanding in this fascinating field. The investigations encompassed a large spectrum of materials studied, which range from weakly localized alloys to strongly localized doped semiconductors, oxides and certain metal-semiconductor composites [2,3]. Recent observation of saturation of dephasing rate of electrons in a disordered system at low temperatures is a new development in this field [4]. In this paper we introduce yet another new dimension to this field of research by presenting some of the important experiments on conductance fluctuation close to the MI transition in doped Si with carrier concentration $n \geq n_{\mathrm{c}}$ (where $n_{\mathrm{c}}$ is the critical concentration of MI transition). There are some past experiments on universal conductance fluctuation (UCF) on disordered (weakly localized) films [5-7]. Systems like carbon com- 
posites and oxide films are also investigated close to MI transition [8,9]. Conductance fluctuation in B-doped single crystalline $\mathrm{Si}$ (with $n \approx 0.8 n_{\mathrm{c}}$ ) has been reported in the temperature range $2 \mathrm{~K}<T<10 \mathrm{~K}$ in order to look for the low frequency charge fluctuation in a Coulomb glass [10]. However, a clean system, like doped $\mathrm{Si}$, has not been investigated close to the MI transition. The motivation of the present investigation is to a carry out a comprehensive investigation of the flicker noise in heavily doped Si over an extensive temperature and frequency range. In this paper we provide a brief resume of some of the important results and discuss the understanding that we have gained from these results.

Noise in a solid can be of three types: thermal noise (with spectral power $=4 k_{\mathrm{B}} T R$ ), shot noise and conductivity noise often known as flicker noise or excess noise. The conductivity fluctuation or the flicker noise often has a power spectra $S_{V} \propto 1 / f^{\alpha}(\alpha \approx 1)$. This noise, popularly known as ' $1 / f$ noise' in condensed matter systems, has been a much-debated issue in the last few decades [11-13]. An understanding of the origin of $1 / f$ noise in a given class of material is important because it has the potential to make noise spectroscopy a useful tool to study the underlying defect dynamics in the solid whose noise is being measured. We show below that investigation of conductance fluctuation and its spectral and temperature dependence gives information which may not be possible to obtain from other measurements. While most other measurements in transport and thermodynamics depend on the average value, noise depends on the statistics of the system which is the source of its uniqueness. The measured noise can be represented by both the spectral power $S_{V}(f)$ or the integrated noise power $\frac{\left\langle(\delta G)^{2}\right\rangle}{G^{2}}=\int \frac{S_{V}(f) \mathrm{d} f}{V^{2}}$. The integration is over the bandwidth of measurement.

Single crystalline $\mathrm{Si}$ without grain boundaries (and well characterized defects) is an attractive clean system to investigate the flicker noise. Noise experiments in heavily doped Si single crystal with carrier concentration $n \geq n_{\mathrm{c}}$ over an extended temperature range has not been done before. All the past studies on doped Si were done for $n \ll n_{\mathrm{c}}$ and in a limited temperature range $[14,15]$. As pointed out before, there is one report of investigation of $1 / f$ noise on B-doped $\mathrm{Si}\left(n \approx 0.8 n_{\mathrm{c}}\right)$ [10]. However, it was done over a very narrow temperature range $2 \mathrm{~K}<T<10 \mathrm{~K}$. We show that when $\mathrm{Si}$ is heavily doped and is very close to the critical region of MI transition, the noise can be substantial. Interestingly, the $f$ and $T$ dependence of the spectral power, $S_{V}(f, T)$ change in a subtle way as the carrier concentration is tuned through the MI transition. However, there is no single physical origin of $1 / f$ noise. Near the MI transition we show that the noise arises from a mechanism like universal conductance fluctuations (UCF) at low temperatures while it arises from classical mechanisms at higher temperatures [16].

\section{Experimental}

We have used Czochralski grown single crystal wafers of Si(111) for the present investigation. The details of the samples are given in table 1. The samples are P doped with $n>n_{\mathrm{c}}$. Disorder was introduced as boron compensation. We have also measured the temperature dependence of resistivity and magnetoresistance (MR) in order to obtain information on the phase breaking length $L_{\phi}$. Transport measurements showed that the samples cover a range from the weak localization (WL) regime to the regime of strong localization (zero temperature conductivity $\sigma_{0} \rightarrow 0$ ). Complete details on the experimental procedures 
Table 1. Physical parameters of the samples used.

\begin{tabular}{lccccc}
\hline Sample & $\begin{array}{c}\text { Doping (P) } \\
\left(\mathrm{cm}^{-3}\right)\end{array}$ & Compensation (B) & $\begin{array}{c}n \\
\left(\mathrm{~cm}^{-3}\right)\end{array}$ & $k_{F} l_{300 \mathrm{~K}}$ & $\sigma_{0}(\mathrm{~S} / \mathrm{cm})$ \\
\hline PS24 & $1 \times 10^{19}$ & 0 & $1 \times 10^{19}$ & 2.8 & $3.1 \times 10^{2}$ \\
PS41 & $6.5 \times 10^{18}$ & 0 & $6.5 \times 10^{18}$ & 2.3 & $1.5 \times 10^{2}$ \\
D150 & $1.67 \times 10^{19}$ & 0.4 & $1.0 \times 10^{19}$ & 1.7 & $1.2 \times 10^{2}$ \\
C286 & $1.1 \times 10^{19}$ & 0.5 & $5.5 \times 10^{18}$ & 0.84 & $\approx 0$ \\
E90 & $1.13 \times 10^{19}$ & 0.6 & $4.5 \times 10^{18}$ & 0.78 & $\approx 0$ \\
\hline
\end{tabular}

can be seen elsewhere $[17,18]$. The noise spectral power in these materials can be low because of the bulk (single crystal) nature of the samples. The samples used had typical thickness of $50 \mu \mathrm{m}$ and width $500 \mu \mathrm{m}$ with an average electrode spacing of $\approx 800 \mu \mathrm{m}$. The active volume for noise determination $(\Omega)$ ranged from $1-50 \times 10^{-6} \mathrm{~cm}^{3}$. Very often noise experiments are carried out in thin films in order to have higher measurable noise. However, one compromises on the quality of the sample. We could measure noise spectral power as low as $\sim 10^{-20} \mathrm{~V}^{2} / \mathrm{Hz}$ using a 5-probe $\mathrm{AC}$ technique [17]. We could also measure down to a frequency of $10^{-2} \mathrm{~Hz}$. This was achieved by high stability in temperature control $|\Delta T| / T<0.01 \%$ and digital signal processing techniques developed in our laboratory [18].

Figure 1 shows $\rho$ vs $T$ data in the temperature range $2 \mathrm{~K}<T<450 \mathrm{~K}$ for some of the samples studied. Important transport parameters like $\sigma(T=0)=\sigma_{0}$ and disorder parameter $k_{F} l$ obtained from experiments are given in table 1 . The disorder parameter (measured at $T=300 \mathrm{~K}$ ) range from $k_{F} l \approx 2.8$ for the metallic (but weakly localized samples) to $\approx 0.78$ for the samples with electron concentration close to the critical concentration and lying in the insulating side. It is evident that three of the samples are metallic with an extrapolated $T=0$ conductivity of $\sigma_{0} \approx 100-300 \mathrm{~S} / \mathrm{cm}$ which is $\sigma_{0} / \sigma_{\text {mott }} \approx 5-15$, where $\sigma_{\text {mott }} \approx 20 \mathrm{~S} / \mathrm{cm}$ is the Mott's minimum metallic conductivity value. Scaling the conductivities with $\sigma_{\text {mott }}$ gives a measure of the distance of the sample from the critical region. Two samples are in the insulating side with $\sigma_{0} \approx 0$.

\section{Results}

We present below a representative cross-section of the noise data. More data and details will be given in the relevant subsections during discussion. A typical example of the variation of noise with frequency is plotted in figure 2. The data shown are typical and shows the magnitude of the spectral power measured. In the same graph we show the background noise which can be measured simultaneously in this technique. The background noise is very close to the thermal noise $\approx 4 k_{\mathrm{B}} T R$. In the inset we show the inverse dependence of the noise on the sample volume $(\Omega)$. This is an important test which establishes that the noise indeed arises from the bulk of the sample. The other inset shows the quadratic dependence of the noise spectral power on the bias $V$ confirming ohmic nature of the noise.

It is customary to normalize the measured $S_{V}(f)$ at each temperature by the relation

$$
\gamma=\frac{f S_{V}(f)}{V^{2}} .
$$

$\gamma$ is closely related to the often used Hoog's parameter [19], $\gamma_{\mathrm{H}}$ by the relation $\gamma_{\mathrm{H}}=n \Omega \gamma$. (Note: $\gamma$ and $\gamma_{\mathrm{H}}$ are frequency independent only when $\alpha$ is strictly equal to 1 . In all 


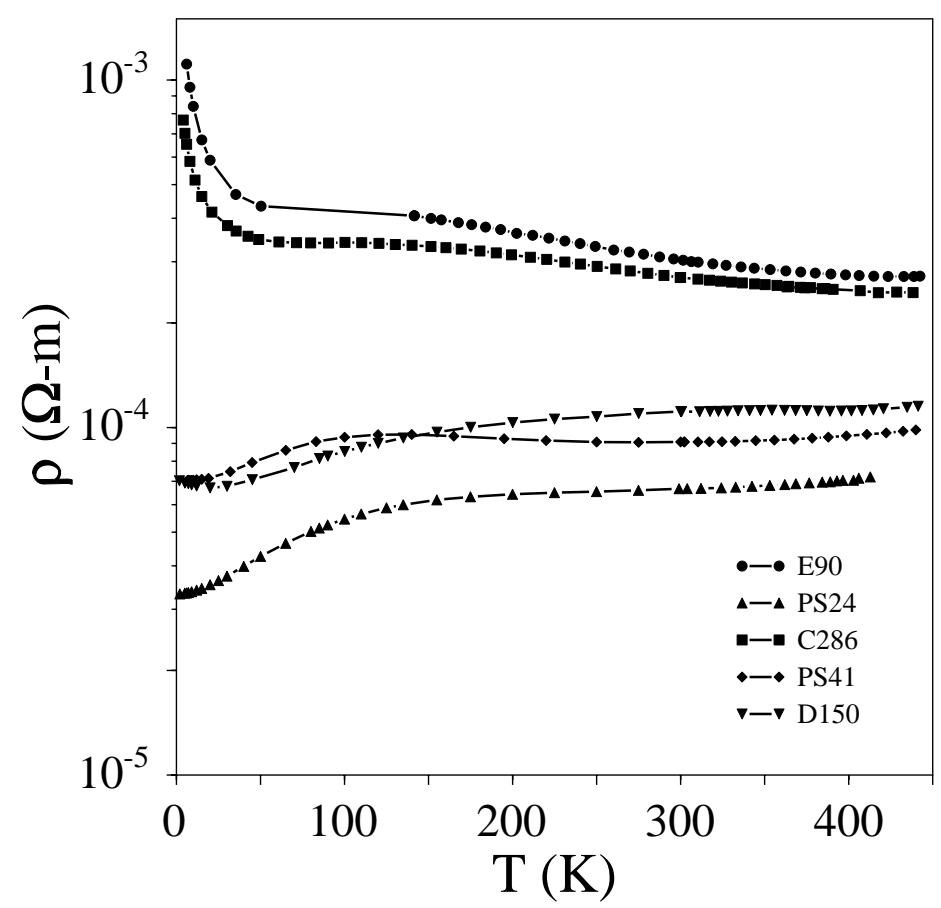

Figure 1. Temperature dependence of resistivity $\rho$ of the Si:P, B samples used. See table 1 for doping level.

other cases it will depend on the measuring frequency.) Figure 3 shows the $T$ dependence of $\gamma_{\mathrm{H}}$ for two typical samples. From figure 3 we can see that there are two distinct temperature regions. There is a high temperature region for $T>200 \mathrm{~K}$, where $\gamma_{\mathrm{H}}$ increases. The temperature dependence of $\gamma_{\mathrm{H}}$ follows an activated behavior. The noise passes through a minima at $T \approx 100-150 \mathrm{~K}$. In the low temperature region for $T<100 \mathrm{~K}, \gamma_{\mathrm{H}}$ rises in both the samples as $T$ is decreased following a relation $\propto T^{-\eta}$. In all the samples studied by us the temperature dependence of the noise shows this behavior (i.e., existence of two temperature regions with a minima at $T \approx 100-150 \mathrm{~K}$ ). However, there are details that change as the carrier concentration changes. We could fit the temperature dependence of the noise over the whole region by the relation

$$
\gamma=\gamma_{0}+\gamma_{\mathrm{LT}} T^{-\eta}+\gamma_{\mathrm{HT}} \mathrm{e}^{E_{\mathrm{A}} / k_{\mathrm{B}} T},
$$

where $\gamma_{0}$ is a temperature independent term and $\gamma_{\mathrm{LT}}$ and $\gamma_{\mathrm{HT}}$ are constants measuring the relative strength of the low and the high temperature contributions respectively. $E_{\mathrm{A}}$ is the activation energy. A typical example of the fit is shown in figure 3. (Note: $\gamma_{\mathrm{H}}$ and $\gamma$ are related by a multiplicative constant only.) Though the behavior can be described by eq. (2) for all the samples, the parameters in the equation change in a distinct manner as the concentration changes and the samples are tuned from the weakly localized region to the 


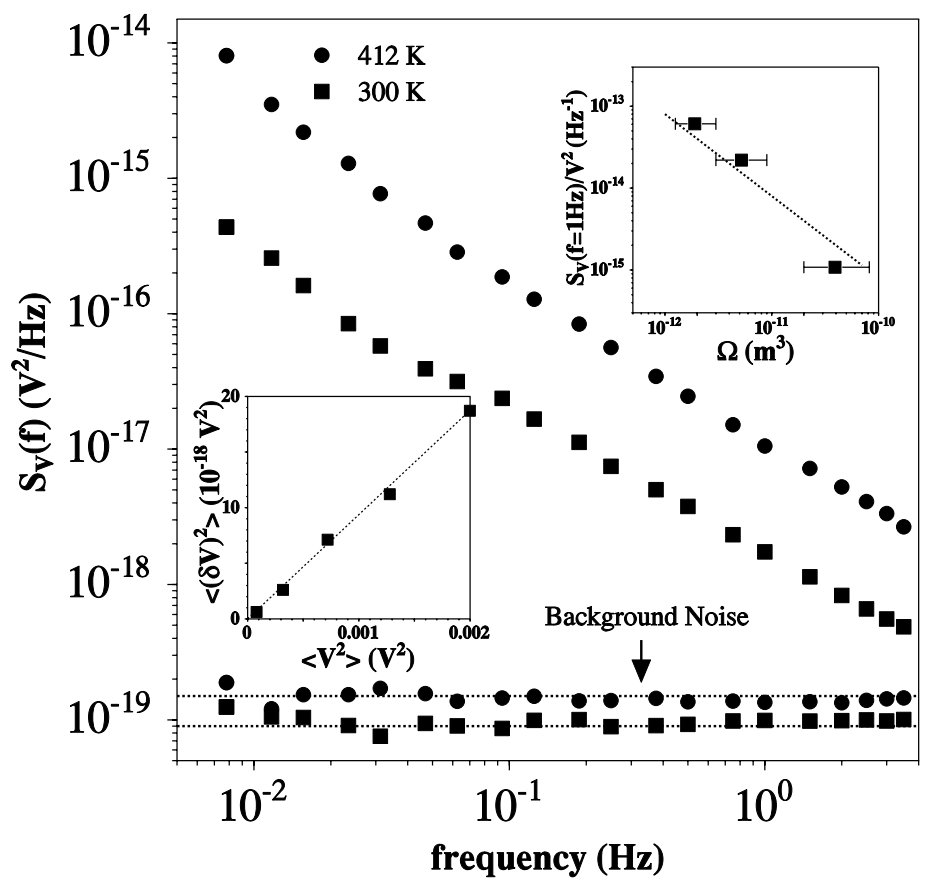

Figure 2. A representative example of the measured power spectra $S_{V}(f)$ as a function of $f$ for two different $T$. The background noise $\left(\approx 4 k_{\mathrm{B}} T R\right)$ are also shown. The inset shows the inverse dependence of the noise on volume which establishes the volume as the source of noise. The other inset shows the quadratic dependence of the spectral power on the bias $\mathrm{V}$.

MI transition. There is a distinct softening of the activation energy $E_{\mathrm{A}}$ as the MI transition is approached. In addition, as MI transition is approached, there is an appearance of the discrete frequency contributions that arise from generation-recombination process. Low temperature dependence described by the parameters $\eta$ also changes as the MI transition is approached from above. $\eta=0.5$ for the weakly localized samples and as the MI transition is approached, $\eta \rightarrow 1.5$. We will show below the temperature dependence of the noise at low temperature (which arises from saturated UCF) mainly determined by the temperature dependence of the phase breaking length $L_{\phi}$. This data will be discussed in detail in the following section.

Existence of two distinct regions of temperature dependence is an interesting result. It clearly shows that we need two distinct mechanisms - one for the high temperature regime and other for the low temperature regime. We will show below that the high temperature regime arises from a classical process and is associated with thermally activated defect dynamics or filling and defilling of electron traps. In contrast, the low temperature regime is a quantum mechanical regime where the phase coherence length of the electrons become important. It arises from UCF. 


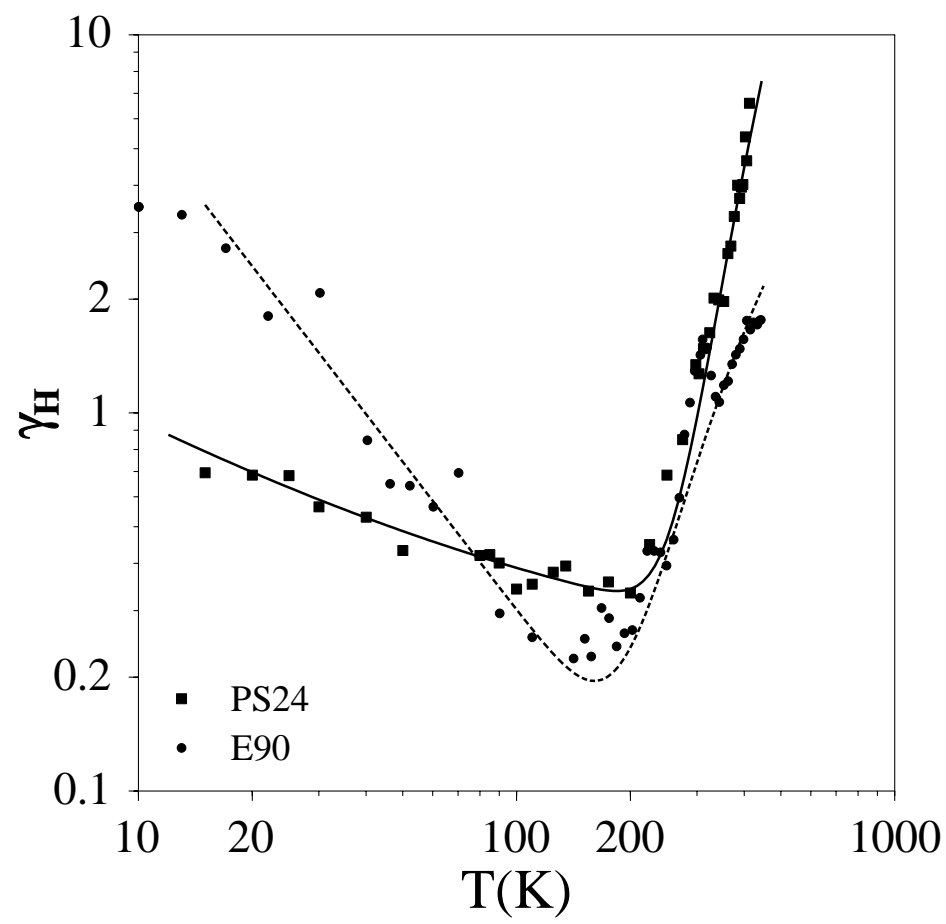

Figure 3. The temperature dependence of the Hoog parameter $\gamma_{\mathrm{H}}$ at a measuring frequency of $1 \mathrm{~Hz}$. The data are for two samples PS24 (metallic) and E90 (insulating).

\section{Discussion}

\subsection{Length scales and crossover from quantum to classical regime}

The transport in a disordered solid like doped $\mathrm{Si}$ (where the disorder causes strong elastic scattering of electrons) is determined by the relative magnitude of elastic mean-free-path $\left(l_{e}\right)$ and the phase breaking length $L_{\phi}(T)$, which is a temperature dependent quantity. As a result it is expected that as the temperature $T$ is changed, the relative ratio of the two length scales will vary. For samples in the WL regime there is a clear theory that allows us to extract $L_{\phi}(T)$ from the negative MR [1]. However, interpreting the MR data is nontrivial because in doped $\mathrm{Si}$ the MR contains a positive contribution from interaction effects in addition to the negative MR due to WL. The positive contribution wins over at the high field region. However, in some of the samples there exists a small window at low temperature and low field which allows a clear determination of $L_{\phi}(T)$. We show the experimental $L_{\phi}(T)$ as a function of $T$ in two of the samples in figure 4 . In the same graph we make the value of $l_{e}$ as determined from $\sigma_{0}$. It can be seen that in the temperature region $T \approx 50 \mathrm{~K}$ they become comparable and for $T<20 \mathrm{~K}, L_{\phi}(T)>l_{e}$, indicating that the transport 


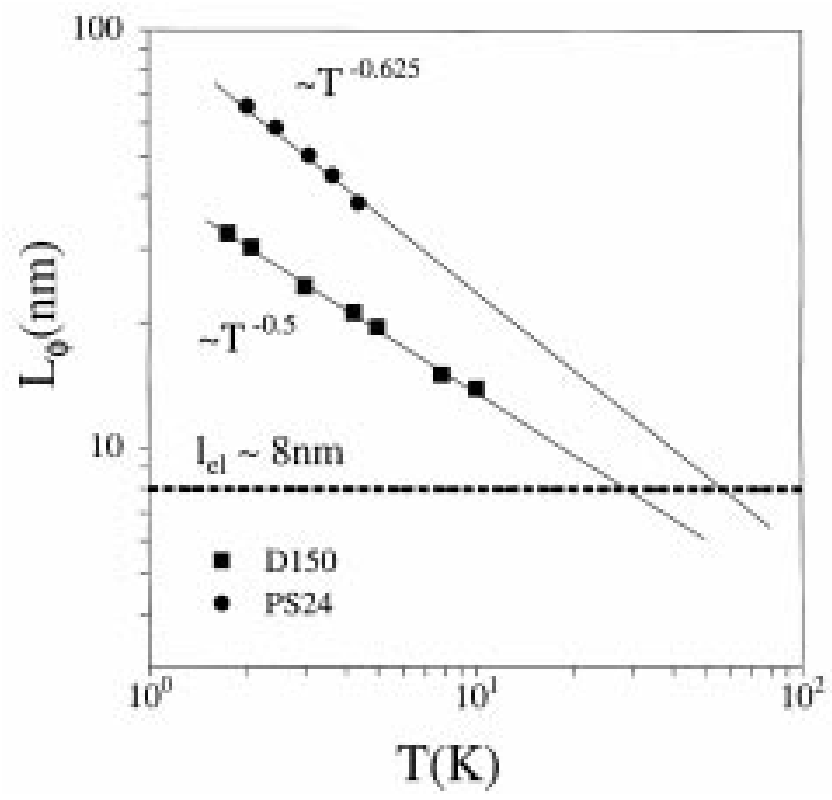

Figure 4. The temperature dependence of the phase breaking length $L_{\phi}$ for two samples PS24 and D150 as obtained from the magnetoresistance data. The elastic mean free path $l_{e}$ is marked for comparison. It can be seen that at $T<50 \mathrm{~K}, L_{\phi}$ is the dominant length scale.

is quantum in nature and is determined by the quantum interference of the backscattered electron waves. The crossover from the low temperature quantum regime to high temperature classical regime which shows up as a minima in $\gamma$, is thus solely determined by the relative strength of $l_{e}$ and $L_{\phi}(T)$.

\subsection{The high temperature classical regime}

In this subsection we focus on the temperature range $T>200 \mathrm{~K}$. We take examples of two samples as case studies. One of the samples, PS24, is in the WL regime and is the most metallic of all the samples $\left(n=2.5 n_{\mathrm{c}}\right)$ with $\sigma_{0} \approx 300 \mathrm{~S} / \mathrm{cm}$ and $k_{F} l \approx 2.8$. The other sample (E90) is close to the critical region and lies in the insulating side (room temperature $k_{F} l \approx 0.78$ ) with $\sigma_{0} \rightarrow 0$. Though in both the samples the noise increase exponentially with temperature as shown in figure $3, E_{\mathrm{A}}$ is much larger for the metallic sample as compared to that of the sample close to the critical region. This exponential dependence of noise with $T$ at higher temperature has been seen in a number of metallic thin films in which the measurements are carried out to high enough temperatures $[11,20]$.

Briefly, the noise (conductivity fluctuation) can arise both from mobility and carrier density fluctuations. For a metallic system the likely source of fluctuation is the mobility fluctuation. In metals the $1 / f$ noise arises from thermally activated relaxation/reorientation of defects which change the scattering cross-sections giving rise to fluctuations in mobility 
and hence the conductivity. In such a system with a distribution of relaxing species and hence a distribution of relaxation time, the phenomenological Dutta-Horn (DH) model seems to provide a basis for the $1 / f$ noise spectral power [11]. The model is based on the generic assumption that the noise arises because the fluctuators have an activated dynamics with a wide distribution of relaxation time. In metallic systems, the broad distribution of relaxation times of statistically independent fluctuators add up to give a featureless $S_{V}(f) \propto 1 / f$ when the distribution of relaxation time $\propto \tau^{-1}$. In this case no discrete characteristic time scales can be seen. According to this model, one can uniquely relate $\alpha$ to the temperature variation of the spectral noise through the relation [11]

$$
\alpha=1-\frac{1}{\ln \left(\omega \tau_{0}\right)}\left[\frac{\partial \ln S_{V}}{\partial \ln T}-1\right],
$$

where $\omega=2 \pi f$ is the measuring frequency and $\tau_{0}$ is an internal time scale for relaxation (jump time). For the two samples in question $\alpha$ was found to vary within the limit 0.9-1.2 and for the metallic sample $\alpha$ has a shallow temperature dependence. We could explain the temperature dependence of $\alpha$ from that of the spectral power dependence $S_{V}$ using eq. (3). This establishes that the high temperature behavior arises from thermally activated defect motion and DH model can provide a quantitative basis for the spectral power dependence.

In the case of the sample lying in the insulating side, in addition to the $1 / f^{\alpha}$ type contribution to the spectral power one observes other features also. $\frac{f S_{V}(f, T)}{V^{2}}$ for the sample as a function of $T$ for different $f$ are shown in figure 5. One can see that there are contributions to the noise at discrete frequencies superimposed on a smooth background. We have analyzed the spectral power $\frac{S_{V}(f, T)}{V^{2}}$ in this sample using the relation

$$
\frac{S_{V}(f, T)}{V^{2}}=\frac{A(T)}{f^{\alpha}}+\sum_{i=1}^{m} \frac{B_{i} f_{i}(T)}{f_{i}(T)^{2}+f^{2}}
$$

where $A(T)$ is dependent only on $T$ and is a measure of the strength of the $1 / f$ fluctuators following the $\mathrm{DH}$ model, as discussed before. $B_{i}$ s are constants which measure the strength of the individual Lorentzian. The $f_{i}(T) \mathrm{s}$ are corner frequencies which are dependent on $T$. Equation (4) was used to fit $\frac{S_{V}(f, T)}{V^{2}}$ for all $f$ and $T$. For the metallic samples all the $B_{i} \mathrm{~s}$ are equal to 0 . For the insulating sample, the corner frequencies of $f_{i}$ s follow a thermally activated behavior with $f_{i}=f_{i 0} \mathrm{e}^{E_{i} / k_{\mathrm{B}} T}$. The activation energies associated with the three $B_{i}$ have values in the range $\approx 0.45-1 \mathrm{eV}$. The existence of discrete peaks in the power spectra strongly suggests existence of generation-recombination (gr) type noise [13] source in this heavily doped sample with $n \leq n_{\mathrm{c}}$ which is lying in the insulating side of the MI transition. The activation energies associated with some of these processes are close to the band gap in $\mathrm{Si}$ and this points to fluctuations arising from excitation across the band gap. Some of the gr process with activation energy $\approx 0.5 \mathrm{eV}$ would imply association of the deep levels.

The main theme of the paper is to investigate what happens to flicker noise in the vicinity of the MI transition. We elaborate this particular aspect in view of the results shown above. In a lightly doped semiconductor the noise arises from both mobility as well as carrier density fluctuations. Defects which create electronic states in the band gap, will contribute to both mobility and carrier density fluctuations predominantly through energy levels that lie close to the Fermi level through a generation-recombination mechanism. This gives 


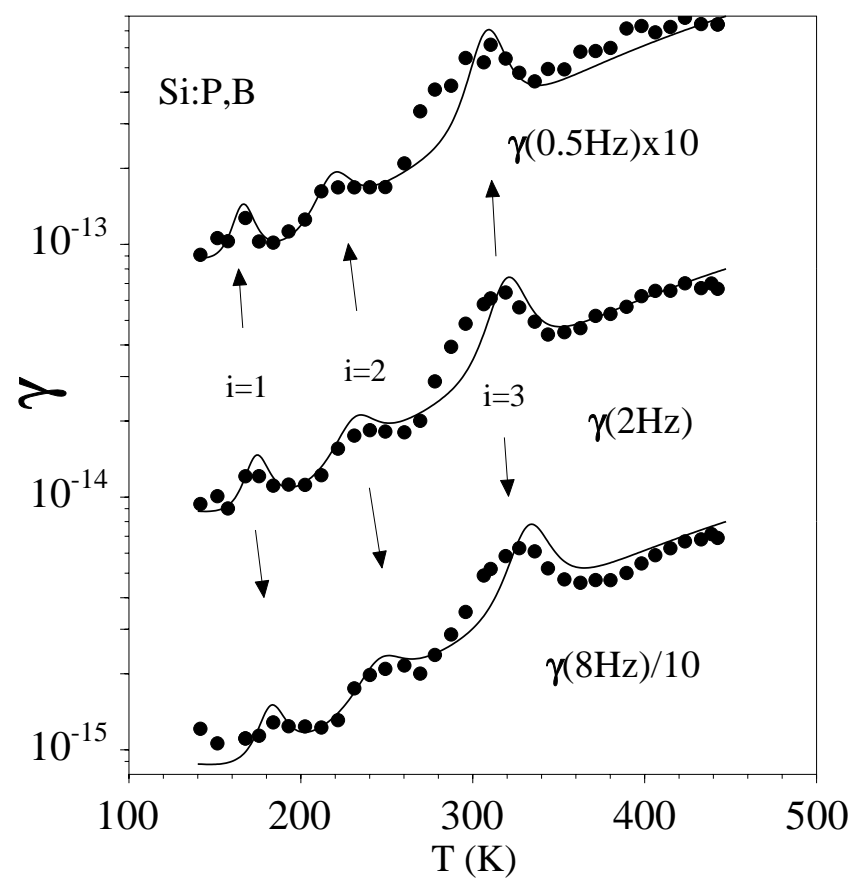

Figure 5. $\gamma$ as a function of $T$ for different $f$ for E90 (insulating). The graph shows the appearance of clear Lorentzian peaks due to the generation-recombination noise (see text).

rise to the Lorentzian contribution to the spectral power at discrete frequencies. However, when the doping level is high $\left(n \geq n_{\mathrm{c}}\right)$, the Fermi level lies inside the conduction band. As a result electronic defect states with energy levels in the gap or in the tail of the conduction band are partially or fully filled and are no longer the dominant sources of $1 / f$ noise. In this case, the noise will originate from mechanisms like that in a metal where the mobility fluctuations arising from defect relaxation dominates. This is the principal conclusion we reach from investigation of the noise at high temperature near the MI transition.

In the metallic regime, as pointed out earlier, mobility fluctuations are caused by changing scattering cross-sections of anisotropic defects as they move/reorient themselves, as envisaged in the 'local interference' (LI) model [20]. In this model it is the anisotropy of the scattering process associated with a reorienting defect complex that leads to conductivity fluctuation. From the observed spectral power we can calculate the integrated noise $\left\langle(\delta G)^{2}\right\rangle$ within the bandwidth of measurement. From this it is possible to estimate the fraction of active sites $n_{m}$ taking part in the noise production by using the relation [20]

$$
N \frac{\left\langle\left(\delta G_{\mathrm{LI}}\right)^{2}\right\rangle}{G^{2}}=\left[n_{\mathrm{Si}} \lambda(T) \beta_{\mathrm{c}} \delta_{\mathrm{c}}\right]^{2} \frac{n_{m}}{n_{\mathrm{Si}}}
$$

where $N$ is the total number of electrons in volume $\Omega, n_{\mathrm{Si}}=5 \times 10^{28} \mathrm{~m}^{-3}$, is the atomic density of $\mathrm{Si}, \beta_{c} \approx 0.20$ is the anisotropy parameter (the value of $\beta$ used is the maximum 


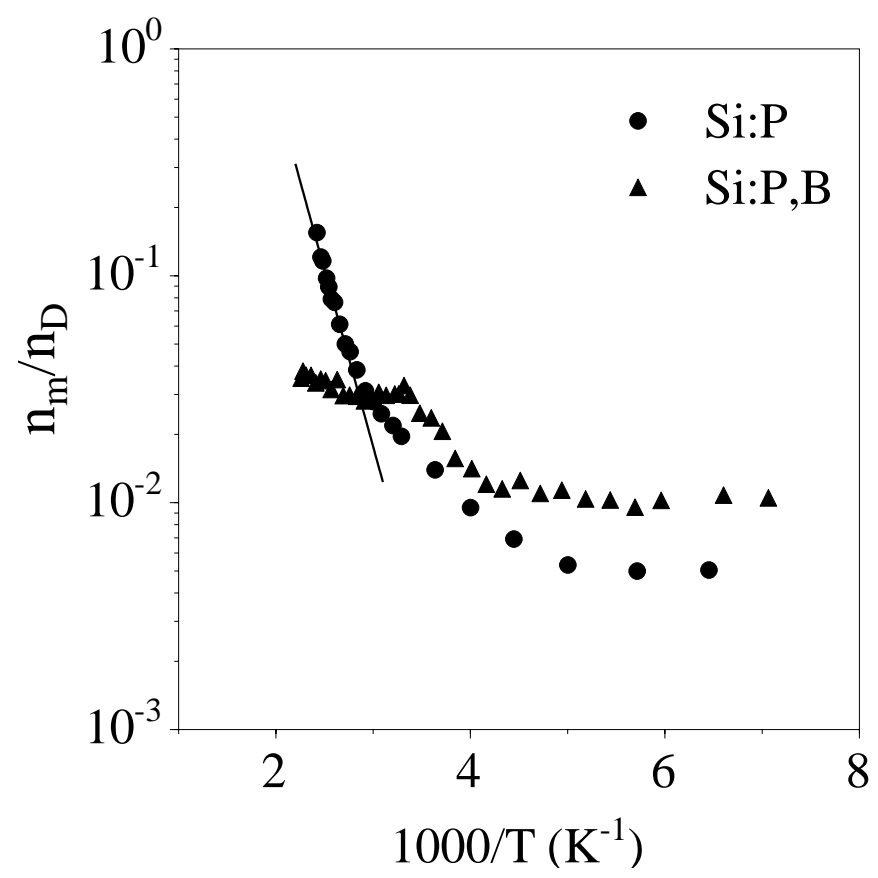

Figure 6. The ratio $n_{m} / n_{D}$ as a function of $T$ as obtained from the local interference model (see text).

that one gets for defect complexes that can give rise to noise in $\mathrm{Si}$ ), $\delta_{\mathrm{c}} \approx 4 \pi / k_{F}^{2}$, the average defect cross-section and $\lambda(T)$ is the net mean free path at temperature $T$. In figure 6 we plot $n_{m} / n_{D}$ which measures the fraction of dopant atoms which are mobile in the time scale $\tau$ that can contribute to the noise at characteristic frequency $f \approx \frac{1}{2 \pi \tau}$. In this particular case, $f$ is the bandwidth of measurement $10^{-2} \mathrm{~Hz}$ to $40 \mathrm{~Hz}$. The plots are for two samples PS24(Si:P) and E90(Si:P,B). We find that within the observed bandwidth at $300 \mathrm{~K}, n_{m} \approx 5 \times 10^{-6} n_{\mathrm{Si}} \approx 0.03 n_{D}$. As $T$ decreases $n_{m}$ also decreases and below $100 \mathrm{~K}$, $n_{m}$ is $\approx 5 \times 10^{-3}-10^{-2} n_{D}$. It can be seen that in Si:P, there is a clear activated behavior of $n_{m}$. Interestingly at lower $T$ in both the samples the ratio $n_{m} / n_{D}$ becomes $T$ independent. It is likely that this is the fraction of mobile defects that take part in noise generation at low temperatures.

\subsection{The low temperature quantum regime}

In the previous subsection we have found that the thermally activated defect motions (both electronic and lattice defects) can explain the noise at high temperature. Below $100 \mathrm{~K}$ when the phase coherence length $L_{\phi}>l_{e}$ the quantum effects like universal conductance fluctuation (UCF) win over. In disordered systems with strong impurity scattering at low temperatures, the noise can arise from the mechanism of UCF [21-23]. At $T=0$ the electrical conductance $(G)$ of a metallic system is an extremely sensitive function of its 
impurity configuration and alteration of position of even a single impurity over a sufficient length scale may induce a conductance change $\delta G_{1} \sim e^{2} / h$. At finite temperatures, the relevant dimension is the regions bounded by electron phase coherence length $L_{\phi}$, within which the interference effects are relevant. Total conductance change due to motions of a number of scatterers inside a region of length scale $L_{\phi}$ is additive (as long as $\delta G_{1} \ll e^{2} / h$ ) and when the number of such scatterers is sufficiently large, UCF noise inside one phase coherent region is saturated with the total variance $\left\langle\left(\delta G_{\phi}\right)^{2}\right\rangle \approx\left(e^{2} / h\right)^{2}$. UCF has been studied extensively in small conductors using magneto-finger-printing. Fluctuations in conductance are recorded as a function of applied magnetic field which changes the phase of the wave function [5]. The UCF, however, can manifest itself in conductors of larger dimensions $\left(L>L_{\phi}\right)$ through temporal conductivity fluctuation which can have a spectral power $1 / f$. In this case the observed magnitude of fluctuations $\left.\left(\langle\delta G)^{2}\right\rangle\right)$ measured on a volume $\Omega$ over the experimental bandwidth, is related to the fluctuation, $\left\langle\left(\delta G_{\phi}\right)^{2}\right\rangle$, in a single phase coherent box of volume $L_{\phi}^{3}$ by the relation [22],

$$
\frac{\left\langle(\delta G)^{2}\right\rangle}{G^{2}}=\frac{L_{\phi}^{3}}{\Omega} \frac{\left\langle\left(\delta G_{\phi}\right)^{2}\right\rangle}{G_{\phi}^{2}} .
$$

In a heavily doped single crystal of $\mathrm{Si}$, the measured noise at low temperatures $(2 \mathrm{~K}$ $<T<100 \mathrm{~K}$ ) and in a magnetic field up to $2 \mathrm{~T}$ indicate that UCF is a predominant source of the observed noise. From the experimental data we also find that the UCF in heavily doped $\mathrm{Si}$ is saturated and this leads to extremely interesting consequences. The confirmatory evidence that the observed noise indeed arises from the UCF mechanism is the sensitivity of $\left\langle(\delta G)^{2}\right\rangle$ to an external magnetic field $H$. The breaking of time reversal symmetry on application of the magnetic field (which introduces an extra phase to the electron's wave function) leads to a reduction of the zero field noise by a factor of 2 at some characteristic field scale $H_{c 1}$. The scale of $H_{c 1} \approx \mathscr{A}(h / e) / L_{\phi}^{2}$, where $\mathscr{A}$ is a constant of the order of unity [22]. In figure 7, we plot the observed variance $\left\langle(\delta G)^{2}\right\rangle$ as a function of $H$ for one of the samples (D150). The reduction of $1 / 2$ occurs very distinctly at field $H_{c 1} \approx 1 \mathrm{~T}$ for low measuring power. Interestingly, the field where the reduction by a factor of $1 / 2$ occurs depends on the measuring power as shown in figure 7 . Observed $H_{c 1}$ increase as the measuring power decreases and eventually $H_{c 1}$ reaches a limiting value of $\approx H=1 \mathrm{~T}$ at $T=2 \mathrm{~K}$. This is an interesting issue and is currently being investigated by us.

In the regime of weak localization one can relate $G_{\phi}$, the conductance in a scale of $L_{\phi}$, to the bulk conductivity $\sigma$ through the relation, $G_{\phi}=\sigma L_{\phi}$. At $T \approx 2 \mathrm{~K}$, for different samples we observed $\left\langle(\delta G)^{2}\right\rangle / G^{2}=(1-3) \times 10^{-13}$. This is indeed a small fluctuation $(<1$ $\mathrm{ppm})$. This also shows why a very high precision noise measurement is needed to study this problem. Using eq. (6) we obtain $\left\langle\left(\delta G_{\phi}\right)^{2}\right\rangle^{1 / 2} \approx(1-1.5) \times\left(e^{2} / h\right)$. Thus, the conductance fluctuations within a single phase coherent region of $L_{\phi}^{3}$ was found to be saturated at $\left\langle\left(\delta G_{\phi}\right)^{2}\right\rangle \approx\left(e^{2} / h\right)^{2}$ at low temperatures. An accurate knowledge of the level of disorder, doping level and also an estimate of the fraction of mobile defects for $T<100 \mathrm{~K}$ (see figure 6) enables us to calculate the change in conductance $\delta G_{1}$ due to movement of a single scatterer. We find $\delta G_{1} \sim e^{2} / h$, which is approximately two orders of magnitude higher than its theoretically expected value in 3D systems [22]. Such a strong coupling 


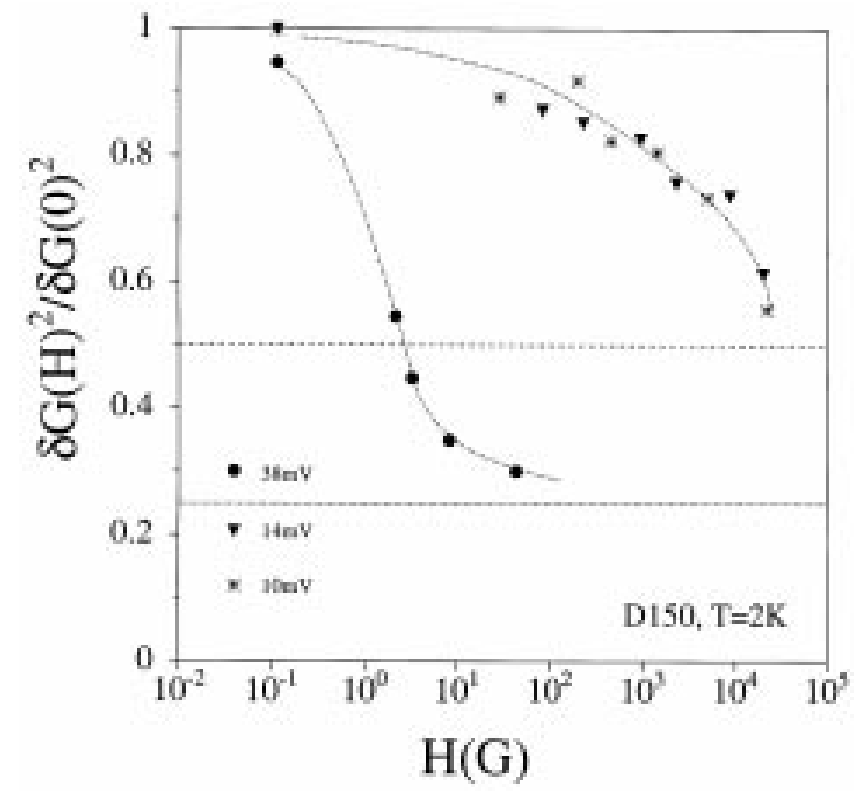

Figure 7. Dependence of the fluctuation on the applied magnetic field (sample D150). Data are shown at two measuring voltages. The drop by a factor of 2 in an applied field is clear.

of defects to electron scattering leads to extreme sensitivity of the conductance to small defect movements and saturation of UCF. This is an important result in the context of $\mathrm{UCF}$ and in doped Si there is a need to reassess some of the fundamental assumptions of the UCF theory. This is in sharp contrast to weakly localized metal films showing UCF where it is not saturated. The saturation of UCF puts an upper limit on the noise magnitude and prevents the noise from diverging at the MI transition. The saturation also leads to important consequences on the temperature dependence of the noise and also on its spectral dependence.

\subsection{Temperature dependence of noise below $100 \mathrm{~K}$}

We have so far established that the noise at low temperatures arises from UCF and that it is saturated. We next show that saturation immediately imposes certain definite constraint on the temperature variation and we experimentally verify this. From eq. (6) we see that for saturated $\operatorname{UCF}\left\langle\left(\delta G_{\phi}\right)^{2}\right\rangle \approx\left(e^{2} / h\right)^{2}$ so that the observed scaled noise, $\left\langle(\delta G)^{2}\right\rangle / G^{2} \approx\left(L_{\phi}^{3} / \Omega\right)\left(e^{2} / h\right)^{2} G_{\phi}^{-2}$. As a result the temperature dependence of the observed noise is governed by the temperature dependence of the combination $\left(L_{\phi}^{3} G_{\phi}^{-2}\right)$. Experimentally, we find that $L_{\phi} \propto T^{-n}$ where $n \approx 0.5-0.6$ (see figure 4). Away from transition, in the WL regime $G_{\phi}$ scales with length $L_{\phi}$ as a result $\gamma_{L} \propto\left\langle\left(\delta G_{\phi}\right)^{2}\right\rangle / G^{2} \propto L_{\phi} \propto T^{-1 / 2}$ or $\eta \approx 0.5$, which is seen experimentally. Interestingly, as the MI transition is approached 
and $G_{\phi}$ becomes scale independent, one expects that the temperature dependence of $\gamma_{L}$ should be determined only by $L_{\phi}$ and $\eta$ should increase. We indeed find that as the MI transition is approached the temperature dependence of $\gamma_{L}$ becomes steeper ( $\eta$ increases). This particular issue needs further investigation particularly below $2 \mathrm{~K}$. Nevertheless, the existing data suggests the observed temperature dependence of the noise $\left(\propto T^{-\eta}\right)$ arises from the saturated nature of the noise.

\subsection{Spectral dependence of noise and electron dephasing by TLS}

The saturated nature of the UCF also leads to interesting constraints on the spectral power $S_{V}(f)$ at low temperatures. In particular, if electron is dephased due to interaction with two-level-systems (TLS), then the number density of TLS puts certain constraints on the spectral power if the noise is due to saturated UCF. This is a fascinating consequence of the UCF theory where number density decides the spectral dependence. Our experiment on doped Si shows that it indeed happens.

The interaction of TLS with electrons have been studied extensively about two decades back in the context of low temperature anomalies in metallic glasses [24,25]. The renewal of interest in electron-TLS interaction originates from the recent experimental observation [4] that the phase coherence time of an electron, $\tau_{\phi}$, shows saturation as $T \rightarrow 0$. Scattering from TLS is being envisaged as a mechanism to dephase electrons at low temperatures so that saturation of $\tau_{\phi}$ can be explained [26,27]. The inelastic electron-TLS scattering rate follows a temperature dependence given as $\tau_{\text {in }}^{-1} \propto T$. Recent work shows that below a certain temperature (similar to 'Kondo temperature' of spin system) the dephasing rate can show a much weaker temperature dependence [27]. The issue if in a metallic wire there are enough TLS to cause the observed rate of dephasing or if the Kondo-like temperature is high enough to make the effects observable close to $T \approx 1 \mathrm{~K}$ have not been settled yet. We have established that dephasing by TLS occurs and it shows up in the noise spectral power. Our low temperature limit $(T>2 \mathrm{~K})$, however, does not allow us to comment on the issue of saturation of the dephasing at low $T$.

The spectral dependence $S(f)$ results from internal dynamics of the electron scattering centers (e.g., lattice defects or impurities) and the system is visualized as a collection of the so-called 'two level states' (TLSs) in the configuration space. Mechanisms like the Dutta-Horn model aim at getting the spectral response like $S_{V}(f) \propto 1 / f$ from a collection of such TLS relaxing through thermal activation. This, however, does not give the absolute magnitude. The evaluation of the absolute magnitude of noise requires a coupling mechanism through which the defect motion gets manifested as the experimentally observed fluctuations in conductance. The theory of UCF provides such a coupling mechanism and can explain the magnitude of the noise. The UCF theory, on the other hand, does not address the issue of the spectral dependence of the noise. This theory generally assumed that if there is a slow dynamics associated with the scatterers with a broad distribution of relaxation time then this can show up as a low frequency $1 / f$ noise. In the following we explore the issue whether the phase coherence of the electronic wave function which is central to UCF has any role in deciding the spectral nature of the noise power whose bare distribution is decided by the TLS nature of the scattering centers. The issue is particularly pertinent because the autocorrelation function for conductance fluctuation due to scattering from a single TLS scatterer may greatly be affected by the phase coherence. 
We consider a collection of TLS with a wide distribution of characteristic flipping rate $f_{\mathrm{c}}$. The electrons scatter inelastically with TLS and each scattering from the TLS scrambles the phase of the incoming electron. In such a situation, the switching of a faster TLS destroys the interference pattern of the electrons considerably, before the switching of a slower TLS occurs. As a result of the preferential role of the fast TLS in affecting the phase of the electron, the decay of the autocorrelation function for the conductance fluctuation from a single TLS scatterer is primarily affected by the fast TLSs present in the system and the fluctuations become less sensitive to the switching of a slow TLS [28]. This insensitivity to slower TLS will lead to flattening of the spectrum at low frequency which will depend on the number density of the TLS $\left(n_{S}\right)$. It can be shown analytically that, starting from a bare spectrum of $1 / f$ form, we get $S(f) \sim f^{-(1-\beta)}$, where $\beta$ is related to the density of active TLS $\left(n_{s}\right)$ through the following relation [28]

$$
\beta \simeq \frac{n_{s} \mathrm{七}_{\phi}^{3} \ln 2}{\ln \left(\frac{f_{\max }}{f_{\min }}\right)} .
$$

In eq. (7), $n_{s}$ is the number of TLS per unit volume, active within the characteristic frequency range of $\left(f_{\max }, f_{\min }\right)$. It is necessary that the bare spectrum has a maximum and minimum frequency in order to have a finite noise power. In the particular case, when the $\mathrm{UCF}$ is saturated it can be shown that $n_{s}$ can be derived as $\left(n_{s} / n\right)_{\text {sat }}=(3 \pi / 2)\left(l / k_{\mathrm{F}} L_{\phi}^{2}\right)$, where $n$ and $k_{\mathrm{F}}$ are the electron density and Fermi wave vector respectively [5]. Rearranging eq. (7) and evaluating the numerical factors, one gets an expression for the ratio of the exponent $\beta$ and the phase coherence length $L_{\phi}$ as

$$
\frac{\beta}{L_{\phi}} \simeq \frac{3 n l}{k_{\mathrm{F}} \ln \left(\frac{f_{\max }}{f_{\min }}\right)}
$$

In figure 8 we show the ratio $\beta / L_{\phi}$ as a function of $T$ for the three samples as measured experimentally. It can be seen that the ratio remains constant as a function of $T$. Interestingly, the experimental determination of the ratio requires data from two completely different set of experiments on the same sample. $\beta$ is obtained from the spectral power density and $L_{\phi}$ from magnetoresistance (MR) experiment. The validity of eq. (8) implies a close relation between the power spectrum and the number density of the TLS through constancy of the ratio $\beta / L_{\phi}$.

\subsection{Dephasing of electron by two-level systems}

The constancy of the ratio $\beta / L_{\phi}$ is based on a very subtle assumption that the electron can dephase by scattering from TLS defects. The experimental result shows that TLS can dephase electrons and thereby determine the spectral dependence of the noise. The conventional wisdom in the field of quantum transport in disordered solids is that the dephasing of electrons that destroy weak localization and weakens UCF arises from sources like electron-electron and electron-phonon scattering. These excitations are 'external' to the static defects which, through strong elastic scattering, cause weak localization. We would like to pose the question: what happens when those defects themselves can dephase 


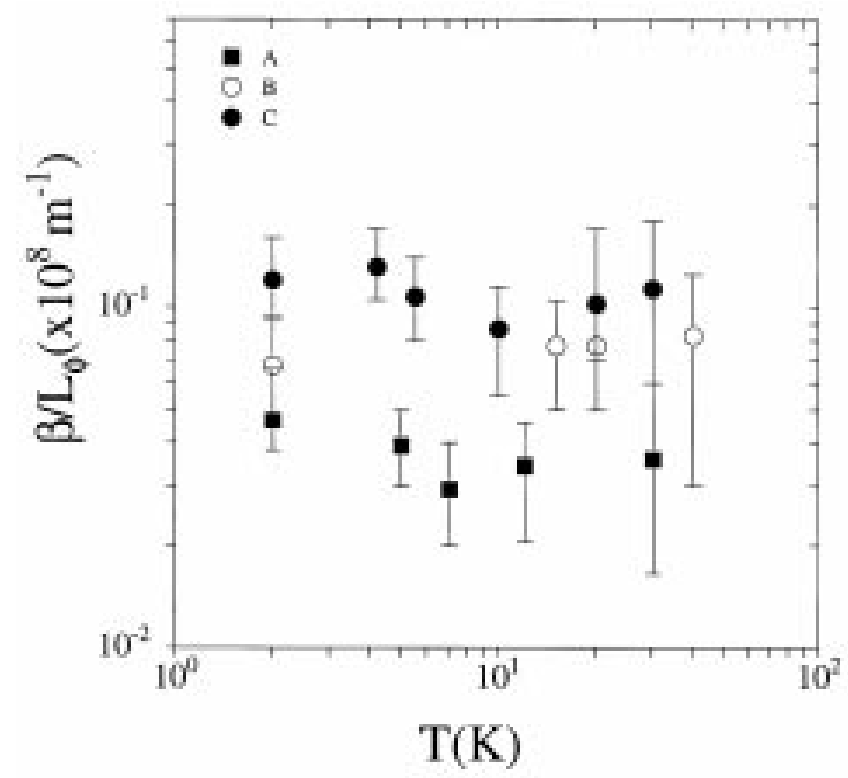

Figure 8. The ratio $\beta / L_{\phi}$ as a function of $T$ as obtained from the noise spectral power and the MR experiment. A(PS24), B(PS41), C(D150). See table 1.

through the mechanism of inelastic scattering. This is a possibility if the defects have an internal structure like TLS associated with it. Our investigation allowed us to probe this issue using saturated UCF as a probe of the dephasing of the TLS associated with dopants like P in single crystalline Si. The observed results that the spectral response of the UCF is modified by the spectrum of flipping rates of the TLS as well as by the number density of TLS, is an independent proof of TLS induced dephasing of electrons.(Note: $L_{\phi} \propto T^{-1 / 2}$ arise from electron-TLS interaction. However, when $L_{\phi}$ and the thermal diffusion length are comparable the latter can also lead to dephasing.)

Our experiment shows that the defects causing the conductance fluctuation are TLStype defects with an internal dynamics. These defects are evidently associated with the dopants $\mathrm{P}$ and $\mathrm{B}$. We may ask the question whether there is evidence that defects in $\mathrm{Si}$ can have TLS-type behavior. Such evidences come from internal friction (low frequency acoustic attenuation) experiments on single crystal Si of comparatively low doping [29]. We are, however, not aware of such experiments done on heavily doped Si. However, if such defects are present for low doping density there is no reason to believe that they will not be present for high doping density. In fact such experiments will be highly desirable and can give direct measurements on the TLS defect densities.

\subsection{Does the noise diverge at the MI transition?}

This is a popular question in the field of MI transition. We argue below that this question cannot be asked in such general terms without further qualification. There are a number 
of issues involved. However, in the context of MI transition in doped Si, it appears that there is no divergence of the magnitude of the noise at finite temperature. We discuss this particular issue in somewhat more details in this subsection. We also discuss our result in the specific context of doped silicon. The noise is measured by the scaled quantity $\gamma$ or $\gamma_{\mathrm{H}}$ and is taken as a measure of the magnitude of noise. The first thing we note is that $\gamma_{\mathrm{H}}$ is temperature dependent and increases both at high and low $T$. At low temperature end where the noise is produced by the mechanism of UCF, the noise cannot diverge because the UCF is saturated at $\left\langle\left(\delta G_{\phi}\right)^{2}\right\rangle \approx\left(e^{2} / h\right)^{2}$. Another effect that can occur for $T<2 \mathrm{~K}$ and can prevent the noise from increasing further at still lower temperature is the saturation of dephasing rate. Since the temperature dependence of noise is indeed dependent on the temperature dependence of $L_{\phi}$, saturation of the temperature dependence of the dephasing rate will lead to flattening of the noise at lower temperature and there will be no divergence.

In the high temperature end the noise arises from classical thermally activated defect dynamics and we can calculate the number of defects involved in the observed noise process using such models as the local interference (LI) model as given by eq. (5). Here also the magnitude of the noise will be limited by the number of available defects that can have a time period of relaxation within the scale of the noise measurements. This is a finite number. Thus the notion that the noise ought to blow up near the MI transition is not a valid one as tested in doped $\mathrm{Si}$. There is no mechanism that will ensure divergence of the noise. One, however, cannot rule out other mechanisms that can cause divergence of the noise near the transition. Though the noise does not blow up near the MI transition, there is enhancement of the noise magnitude in heavily doped Si. For instance, in lightly doped $\mathrm{Si}\left(n \leq 10^{-16} \mathrm{~cm}^{-3}\right)$ the value of $\gamma_{\mathrm{H}}$ is typically $\leq 10^{-2}$ irrespective of whether the noise is from the bulk, surface or interface. In disordered metallic films which are in the weakly localized regime the typical $\gamma_{\mathrm{H}} \leq 10^{-3}$. In contrast to doped Si we see $\gamma_{\mathrm{H}} \rightarrow 1-10$ at $T=2 \mathrm{~K}$. This is definitely high but is not a divergence. Interestingly in two other systems $\mathrm{C}-\mathrm{Cu}$ films [8] and films of an oxide system $\mathrm{La}_{1-x} \mathrm{Sr}_{x} \mathrm{VO}_{3}$ [30] the $\gamma_{\mathrm{H}}$ does not diverge but reaches a limiting value in the vicinity of $1-10$. The only material where a diverging $\gamma_{\mathrm{H}}$ at room temperature is reported is the $\mathrm{In}_{2} \mathrm{O}_{x}$ film near the verge of MI transition [9]. To summarize, we find clear evidence that the noise is large near the MI transition but it does not diverge.

\section{Conclusion}

In this paper we have presented a brief summary of the important results that we have obtained from our investigation of flicker noise in heavily doped Si close to the MI transition. Our result clearly shows some of the basic mechanisms that are involved. We stress that a quantitative analysis of the fluctuation is needed to understand the atomic process involved in generating the noise.

\section{Acknowledgement}

One of us (AKR) wishes to thank CSIR, Government of India, for a sponsored project. SK wishes to thank CSIR for an SRF. 


\section{References}

[1] P A Lee and T V Ramakrishnan, Rev. Mod. Phys. 57, 287 (1985)

[2] P P Edward and C N R Rao, The metallic and nonmetallic states of matter (Taylor and Francis, New York, 1995)

[3] A K Raychaudhuri, Adv. Phys. 44, 21 (1995)

[4] P Mohanty, E M Q Jariwala and R A Webb, Phys. Rev. Lett. 78, 3366 (1997)

[5] N Giordano, in Mesoscopic phenomena in solids edited by B L Altshuler, P A Lee and R A Webb (Elsevier, New York, 1991)

[6] N O Birge, B Golding and W H Haemmerle, Phys. Rev. B42, 2735 (1990)

[7] J S Moon, N O Birge and B Golding, Phys. Rev. B56, 15124 (1997)

[8] G A Garfunkel, G B Alers, M B Weissman, J M Mochel and D J VanHarlingen, Phys. Rev. Lett. 60, 2773 (1988)

[9] Z Ovadyahu, Phys. Rev. Lett. 52, 569 (1984)

[10] J G Massey and M Lee, Phys. Rev. Lett. 79, 3936 (1997)

[11] P Dutta and P M Horn, Rev. Mod. Phys. 53, 497 (1981)

[12] M B Weissman, Rev. Mod. Phys. 60, 537 (1988)

[13] B K Jones, Adv. Electron. Electron Phys. 87, 201 (1994)

[14] T Kuhn, L Reggiani, L Varani and V Mitin, Phys. Rev. B42, 5702 (1990)

N V Dyakonova, M E Levinshtein and S L Rumyantsev, Sov. Phys. Semiconductor 25, 1241 (1991) and references therein

[15] L Michelutti, Phys. Rev. B57, 12360 (1998)

[16] A Ghosh and A K Raychaudhuri, Phys. Rev. Lett. 84, 4681 (2000)

[17] J Scofield, Rev. Sci. Instrum. 58, 985 (1987)

[18] A Ghosh, Ph.D. Thesis, Indian Institute of Science, Bangalore (1999)

[19] F N Hoog, Physica B83, 14 (1976)

[20] For example, J Pelz and J Clarke, Phys. Rev. B36, 4479 (1987)

[21] S Feng, P A Lee and A D Stone, Phys. Rev. Lett. 56, 1960 (1986)

[22] P A Lee, A D Stone and H Fukuyama, Phys. Rev. B35, 1039 (1987)

[23] A D Stone, Phys. Rev. B39, 10736 (1989)

[24] J L Black, B L Gyorffy and J Jäckle, Philos. Mag. B40, 331 (1979)

[25] H Hunklinger and A K Raychaudhuri, Progress in low temperature physics (1986) Vol. IX, p. 265

[26] Y Imry, H Fukuyama and P Schwab, Europhys. Lett. 47, 608 (1999)

[27] A Zawadowski, Jan von Delft and D C Ralph, Phys. Rev. Lett. 83, 2632 (1999)

[28] M B Weissman, Phys. Rev. Lett. 59, 1772 (1987)

[29] R N Kleiman, G Agnolet and D J Bishop, Phys. Rev. Lett. 59, 2079 (1987)

[30] E Prasad, M Sayer and J P Noad, Phys. Rev. B19, 5144 (1979) 